\title{
Historia de las disciplinas, profesionalización docente y formación de profesores: el caso español ${ }^{1}$
}

\author{
Antonio Viñao*
}

\section{Resumen}

Este texto pretende analizar, a partir del caso español, las rela-

* Universidad de Murcia,

ciones existentes entre la historia de las disciplinas (y, dentro de España.avinao@um.es

ella, de los manuales escolares), el proceso de profesionalización docente y la formación de profesores. Primero se exponen las causas que explican la ausencia legal, en el caso español, de la historia de las disciplinas en la formación de profesores, así como algunas iniciativas aisladas en relación con este tema. Seguidamente se da cuenta de algunos aspectos o cuestiones en los que fundamentar la presencia de la historia de las disciplinas en la formación de profesores, en especial de la necesidad de insertar los estudios sobre la manualística escolar en el ámbito, más amplio, de dicha historia. Por último, se exponen algunos conceptos e ideas clave para estructurar la historia de las disciplinas.

\section{Palabras clave}

disciplinas escolares; profesionalización docente; formación de profesores; manuales escolares; código disciplinar.

1. Este texto está financiado por la Fundación SénecaAgencia de Ciencia y Tecnología de la Región de Murcia en el marco del II PCTRM 2007-2010, dentro del proyecto de investigación sobre "El patrimonio histórico-educativo de la Región de Murcia. La memoria de los docentes". 


\title{
History of school subjects, the professionalization of teachers and teachers' training: the Spanish case
}

\begin{abstract}
This text tries to analyze, through a Spanish case, the relationships existing among the history of school subjects (and, inside it, the history of textbooks), the process of the professionalization of teaching and teachers' training. First, the causes explaining the legal absence, in Spanish, of the history of school subjects in teachers' training, as well as some isolated initiatives on this theme are presented. Then, some aspects and issues are dealt with in order to justify the presence of the history of school subjects in teachers' training, especially the need to include the analysis of textbooks in the wider field of such history. Finally, some key concepts and ideas with the aim of shaping the history of the school subjects are also presented.
\end{abstract}

Key words schoolsubjects; teachers'professionalization; teachers'training; textbooks; school subject code. 
A diferencia de lo que acaece en Francia, donde dos recientes órdenes ministeriales de 28 de diciembre de 2009 han introducido la historia de las disciplinas escolares en las pruebas de los concursos de acceso al profesorado de educación secundaria; donde, bajo el patrocinio del Service d’Histoire de l’Éducation, se han promovido seminarios y encuentros sobre el tema; y donde el desarrollo de la historia de la enseñanza de cada disciplina ha tenido lugar a demanda de los docentes de las mismas (Caspard, 1998; Julia, 2000), en el caso de España no puede decirse que la historia de las disciplinas en general, o de alguna de ellas en particular, forme parte, desde un punto de vista legal, de la formación inicial o en ejercicio de los profesores, o que su desarrollo, salvo excepciones, se haya producido a causa de la demanda de quienes las imparten. En lo que a la formación inicial se refiere, no existe en la normativa vigente materia alguna de este tipo en el grado que habilita para el acceso a la docencia, tanto en el caso de la educación infantil o primaria como en el de la secundaria. Tampoco la hay en el máster psico-pedagógico, teórico y práctico, que se exige para poder presentarse a los concursos de acceso al profesorado de educación secundaria. Por lo que respecta a la formación oficial en ejercicio, en manos desde 1983 de los Centros de Profesores y Recursos, la presencia de cursos o seminarios sobre la historia de las disciplinas ha sido algo muy excepcional, por no decir inexistente.

Eso no quiere decir, como se verá, que no existan posibilidades dentro del actual marco legal, propuestas o incluso alguna iniciativa o experiencia en relación con la introducción de la historia de las disciplinas en la formación de profesores.

\section{Causas explicativas de dicha ausencia legal}

Las causas explicativas de esta ausencia son varias y todas ellas se apoyan y refuerzan. Desde un punto de vista tanto individual como corporativo, la memoria histórica de la educación entre los profesores no suele ir más allá del momento en que accedieron como tales al sistema educativo o, en todo caso, de aquella época o tiempo que recuerdan como alumnos. Su memoria como profesores se limita, además, al campo o área disciplinar a que pertenecen y al nivel educativo en el que ejercen la docencia. En síntesis, los docentes, como cualquier otro grupo profesional, tienden a considerar: (a) que la historia de la educación comienza con su experiencia -memoria-personal y profesional de la misma y que antes de dicha experiencia sólo existe un magma más o menos confuso e invariable; (b) que dicha historia se centra o gira en torno a su campo disciplinar y al nivel educativo al que pertenecen; y (c) que cual- 
quier tiempo pasado -en especial aquél en el que cursaron sus estudios u otro tiempo mítico no concretado- fue mejor que el actual en lo que a la educación se refiere. Su memoria de la educación -una memoria limitada no sólo en el tiempo sino también en el espacio académico- es una combinación de memoria personal -por tanto generacional- y corporativo-profesional. Hacen de la memoria historia, que es algo diferente, y de su entorno disciplinar y académico un mundo cerrado a otros. No advierten, salvo excepciones, que la configuración actual de su nivel o modalidad de enseñanza y de su campo disciplinar, aquél que les proporciona su identidad como profesores, es el resultado de un dilatado proceso de construcción social y cultural cuya duración excede de su temporal y profesionalmente limitada experiencia personal. Como tampoco advierten, por lo general, que existen fuertes similitudes, y algunas diferencias, entre los procesos de disciplinarización o asignaturización de cada una de las materias, actividades o áreas disciplinares. Es decir, que dichas materias y áreas forman parte de un sistema educativo que genera su propia cultura y subculturas, dentro del cual se producen una serie de procesos y actúan una serie de fuerzas internas y tendencias que condicionan la configuración actual tanto del nivel educativo como del campo disciplinar en el que ejercen la docencia. Y que, por tanto, es posible llevar a cabo un análisis general de los distintos procesos de disciplinarización que vaya más allá del nivel o campo que les define como profesores (Viñao, 2002, 2006a y 2006b).

Una segunda causa reside en la concepción que usualmente se tiene de la historia como un campo inagotable de curiosidades y anécdotas, como un lugar de citas, referencias, acontecimientos y personajes, que conmemorar u olvidar. La historia como un cúmulo de procesos de larga duración, de genealogías, de capas superpuestas y palimpsestos - la historia como ciencia-, es algo normalmente ajeno a la mentalidad de las gentes. Si resulta, además, que en vez de ofrecer recetas, respuestas y soluciones prácticas de aplicación inmediata a los problemas cotidianos del profesor en el aula, lo que la historia de las disciplinas ofrece son preguntas y planteamientos que obligan a reflexionar, a pensar, y que socavan los cimientos del relativo saber-poder en que se asienta la labor docente en un área o campo determinado, al mostrar lo que de construcción social hay en el mismo y en la docencia como profesión, nada tiene de extraño que los profesores rechacen sus planteamientos o análisis. No hay grupo profesional que soporte psicoanalizarse como tal grupo desvelando los obscuros, humildes o conflictivos orígenes de los que proceden sus saberes-poderes, o mostrando cómo, en el fondo, dichos saberes y poderes se asientan sobre una determinada com- 
binación histórica de grandes ideales, más o menos retóricos, e intereses individuales y corporativos. Cuando el pasado no resulta complaciente, mejor es ignorarlo creando todo tipo de leyendas y mitos sobre el mismo, o modificarlo a nuestro gusto y antojo.

Una tercera causa, consecuencia de las dos anteriores, se halla en el escaso desarrollo de la investigación sobre la historia de las disciplinas. Si no se investiga sobre un tema, eso quiere decir que dicho tema no se considera relevante y que no interesa. Y si no se investiga sobre el mismo, mal puede pensarse que su presencia en la formación de profesores sea necesaria. Entre otras razones, porque se desconoce la existencia de este campo de investigación, así como lo que puede aportar al conocimiento y a la reflexión sobre la docencia como profesión. El cuadro que a continuación se incluye, muestra la presencia en España, en porcentajes, de artículos de historia de las disciplinas en las principales revistas de Historia de la Educación, Historia de la Ciencia, Didáctica general y Didácticas específicas desde el año 2000 al 2009, ambos inclusive.

\section{Presencia de la historia de las disciplinas en diversas revistas}

(\% de artículos sobre el total en el período 2000-2009)

\begin{tabular}{|l|c|}
\hline Con-Ciencia Social. Revista del grupo Fedicaria & 48.72 \\
\hline Llull. Revista de la Sociedad Española de Historia de las Ciencias y de la Técnica & 18.95 \\
\hline Historia de la Educación. Revista de la Sociedad Española de Historia de la Educación & 15.40 \\
\hline Didáctica de las Ciencias Experimentales y Sociales & 10.81 \\
\hline Iber. Didáctica de las Ciencias Sociales & 5.31 \\
\hline Enseñanza de las Ciencias. Revista de Investigación y Experiencias Didácticas & 4.17 \\
\hline Suma. Revista sobre el aprendizaje y la enseñanza de las Matemáticas & 1.88 \\
\hline Eufonía. Didáctica de la Música & 1.79 \\
\hline Profesorado. Revista de Currículum y Formación de Profesorado & 1.76 \\
\hline Alambique. Didáctica de las Ciencias Experimentales & 1.53 \\
\hline Tándem. Didáctica de la Educación Física & 1.09 \\
\hline Uno. Didáctica de las Matemáticas & 0.77 \\
\hline Textos. Didáctica de la Lengua y la Literatura & 0.67 \\
\hline
\end{tabular}

Que la revista Con-Ciencia Social del grupo Fedicaria, dedicada a la Didáctica de las Ciencias Sociales -geografía e historia preferentemente- alcance un porcentaje tan elevado (48.72 \%) no debe extrañar si se tiene en cuenta: (a) el bajo número de ar- 
tículos por número (entre 3 y 6), y el hecho de que en cada uno de ellos se incluye un análisis bioprofesional de un profesor y una entrevista con el mismo, lo que supone ya, al menos, dos textos sobre el tema que nos ocupa; y (b) que es en Fedicaria (http:// www.fedicaria.org) y en especial, aunque no sólo, en el subgrupo Nebraska que forma parte del mismo (http://www.nebraskaria.es) donde mayor desarrollo teórico, conceptual y productivo ha tenido la historia de las disciplinas escolares en España.

Los porcentajes de Llull (18.95 \%) e Historia de la Educación (15.40 \%) son lo menos que cabe esperar de dos revistas dedicadas, respectivamente, a la historia de la ciencia y a la de la educación. Las cifras de las revistas de las didácticas específicas -o de didáctica general como Profesorado-, contrastan con las hasta ahora indicadas. Por lo general no superan el 2 por ciento y, cuando lo hacen, es porque la mayor parte de los artículos sobre el tema ( 5 de un total de 8 en Didáctica de las Ciencias Experimentales y Sociales) están escritos por miembros del grupo Fedicaria; porque, en el caso de lber, hay dos números monográficos de índole bioprofesional (los dedicados a dos historiadores tan relevantes como P. Vilar y A. Domínguez Ortiz); o porque, como sucede en Enseñanza de las Ciencias, se trata de trabajos sobre historia de los manuales escolares o de historia de la ciencia con estrechas relaciones con su enseñanza.

Queda asimismo, como hecho a destacar, que ninguna de las revistas indicadas -ni siquiera Llull o Historia de la Educación- ha publicado un número monográfico sobre la historia de las disciplinas escolares a lo largo de los nueve años consultados, ni en alguno de los números anteriores. Un análisis de los monográficos y de los títulos de los trabajos incluidos en las revistas de didácticas específicas nos muestra una serie de intereses o temas comunes o recurrentes en todas ellas -atención a la diversidad, competencias a desarrollar en los alumnos, recursos didácticos, materiales curriculares, nuevas tecnologías, formación de profesores, procesos de enseñanza y aprendizaje, estrategias de actuación en el aula, experiencias, actividades e innovaciones, leyes o reformas en curso,...- entre los que no se halla la historia o socio-génesis de la enseñanza

2. Un caso paradigmático sería el de la revista Suma de didáctica de las matemáticas. En su página web se ofrece una clasificación de los artículos publicados en la misma agrupados en 27 epígrafes o temas entre los que no aparece la historia de la enseñanza de las matemáticas, es decir la historia del campo disciplinar que constituye el objeto de la revista. Los escasos artículos aparecidos sobre el tema ( 9 entre 480 del año 2000 al 2009) se incluyen en el epígrafe "Historia de las matemáticas". de la disciplina o campo disciplinar en cuestión; es decir, cómo nació y cómo ha llegado a configurarse la disciplina o campo en el que se trabaja. ${ }^{2}$ Esta es una cuestión que ni interesa ni preocupa, salvo cuando se refiere a la evolución reciente de dicha disciplina o campo -nunca suele irse más allá de la memoria profesional de quienes escriben-, o uti- 
lizando un marco teórico-conceptual que sitúe dicha evolución en el tiempo y en el espacio social y académico correspondiente. En síntesis, como ya se ha señalado, la visión temporal del campo profesional en el que se insertan dichos trabajos se limita, como mucho, a la vida profesional de sus autores, y su visión socio-espacial, como también es habitual, se circunscribe al espacio acotado de su disciplina.

Por último, formación, docencia e investigación se refuerzan. No se investiga, como acaba de verse, sobre aquello que no se considera relevante en la formación de profesores. Y tampoco se forma en aquello que no se considera digno de ser enseñado o investigado. Estamos ante un círculo vicioso que la misma historia de las disciplinas ilustra y aclara. Al fin y al cabo se trata de configurar un nuevo campo disciplinar, el de la historia de las disciplinas, que entra en conflicto con los ya existentes en la formación de profesores y que si ha de ocupar un espacio en los grados o másteres de formación de profesores tiene que ser a costa del tiempo de formación dedicado a otras materias o disciplinas, a otros campos con sus correspondientes protagonistas e intereses corporativos e individuales. Uno de los problemas de la historia de las disciplinas es que se trata de un campo interdisciplinar. Un campo en el que necesariamente tienen que trabajar y colaborar investigadores y formadores que proceden de campos disciplinares ya constituidos, cuyos colegas ven la historia de las disciplinas como un nuevo campo competidor a la hora de ocupar el espacio académico, necesariamente limitado, de la formación de profesores. Ello explica que los ejemplos que en España pueden ponerse en relación con la introducción de la historia de las disciplinas en la formación de profesores hayan tenido su origen en iniciativas dispersas y locales llevadas a cabo por quienes se interesan por estos temas, y que su continuidad en el tiempo e importancia cuantitativa -medida por el número de horas o créditos que se le dedican-esté ligada a la presencia de dichos profesores, y a su peso o relevancia en las instituciones responsables de dicha formación. En síntesis, la presencia de la historia de las disciplinas en la formación de profesores depende de la existencia o no, en una institución formativa determinada, de una persona o un grupo de personas que investiguen en este campo y de su peso o grado de influencia en dicha institución.

\section{La historia de las disciplinas y la formación de profesores en España: algunas iniciativas aisladas}

La ausencia de un mandato legal en relación con la introducción en España de la historia de las disciplinas en la formación de profesores no significa que no existan, 
o hayan existido, iniciativas aisladas en tal sentido. Como no podía ser de otro modo, dichas iniciativas proceden de quienes, desde diferentes campos académicos-Historia de la Ciencia, Historia de la Educación, Didácticas específicas- han investigado o investigan en el ámbito de la historia de las disciplinas. Además, como se ha dicho, dicha presencia depende no sólo de que en una institución docente determinada, y en un determinado momento, haya quien o quienes investiguen en este campo, sino también de la capacidad de dichas personas, por su poder e influencia en las referidas instituciones y por las posibilidades que el marco legal establece, para introducir y hacerse cargo de la enseñanza y formación en el ámbito de la historia de las disciplinas.

Así, los casos de los que se ha podido reunir información se refieren a las universidades de Murcia -Licenciatura de Pedagogía y Máster de Secundaria-, Oviedo -Licenciatura de Pedagogía-, Salamanca -Diplomatura de Maestro de Primaria, Máster de Secundaria, Curso de Posgrado de Didáctica de Historia, Geografía y otras Ciencias Sociales-, Santander -Grado de Maestro de Primaria, Máster de Secundaria-, Universidad Nacional de Educación a Distancia -Máster de Innovación e Investigación en Educación-y Valencia -Máster de Historia de la Ciencia y de la Comunicación Científica-. Dicho esto, conviene advertir que:

- En algunos casos -diplomaturas y licenciaturas- se trata de titulaciones a extinguir sin que en los nuevos grados, que vienen a sustituirlas, figure la historia del campo disciplinar o de las disciplinas entre las asignaturas a impartir, siquiera como un tema o parte de las mismas.

- Hay amplias diferencias en lo que significa la presencia de la historia de las disciplinas en función de quienes se hacen cargo de ella y de los destinatarios: (a) en unos casos se ofrece una perspectiva general sobre este campo de investigación; (b) en otros la formación se centra en una disciplina concreta como, por ejemplo, la Didáctica de las Matemáticas o la de la Geografía e Historia; (c) en otros, se liga al estudio de la cultura material de la educación científica y a la conservación del patrimonio científico-educativo; (d) en otros, por último, su estudio se plantea junto con el de la cultura escolar y de los libros de texto en relación con los procesos de innovación educativa, o con enfoques genealógico-disciplinares ligados al pensamiento histórico del presente.

- Dentro del reducido número de horas o créditos dedicados al tema en todos los casos, existen asimismo notables diferencias en este punto entre ellos.

En relación, pues, con el nuevo máster para los futuros profesores de educación 
secundaria, aquellos casos conocidos en los que, siquiera de un modo testimonial o mínimo, se incluyen cuestiones relativas al ámbito de la historia de las disciplinas, muestran que dicha presencia se produce en aquellas pocas universidades en las que existen profesores que investigan en este campo. Un análisis más general podría hacerse a través de los libros y materiales que se están comenzando a editar para dicho máster. Un buen ejemplo, de una editorial muy conocida en el ámbito de la pedagogía -la editorial Graó de Barcelona-, sería su nueva colección "Formación del profesorado. Educación Secundaria” cuyos libros, hasta un total de 49, han aparecido a comienzos del curso 2010-11. En algunos campos disciplinares (Biología y Geología, Física y Química) se intenta relacionar, siquiera de un modo sumario, la historia del campo científico correspondiente con el de su enseñanza. En otros (Dibujo, Educación Física, Geografía e Historia, Inglés) se incluye un capítulo sobre la historia de su enseñanza, que, por lo general, intenta conectar su pasado con la situación actual o más reciente que es lo que realmente interesa. En otros (Filosofía, Francés, Lengua Castellana y Literatura, Lengua Catalana y Literatura, Matemáticas, Música, Tecnología, Orientación Educativa) no se hace referencia alguna al tema.

\section{Aspectos y cuestiones en los que fundamentar y plantear la presencia de la historia de las disciplinas en la formación de profesores}

Toda introducción en los planes de estudio de una materia o tema novedoso requiere el apoyo de una serie de argumentos acerca de su utilidad y objetivos y, por supuesto, una estrategia dirigida a tal fin. En este sentido, las líneas que siguen pretenden mostrar algunos aspectos, temas o cuestiones en los que fundamentar y apoyar la presencia de la historia de las disciplinas en la formación de profesores.

Junto a la categoría o cuerpo al que se pertenece y la institución en la que se prestan servicios, los dos principales aspectos que definen la identidad de los profesores son el nivel o modalidad educativa en la que se imparte la docencia y la disciplina o campo disciplinar al que se está adscrito. Tanto uno como otro aspecto nos remiten al proceso de profesionalización docente, a las culturas escolares y académicas -tradiciones, rituales, rutinas, modos de hacer, de pensar, de manejar el aula y de enseñar, sedimentados a lo largo del tiempo y transmitidos de una generación a otra de profesores- y, en relación con ellos, a la historia de las disciplinas o actividades escolares, ya se trate de las matemáticas o de la historia en la educación secundaria, 
la ortografía, el dictado o el cálculo en la educación primaria, o el derecho penal en la enseñanza universitaria. No es posible entender el proceso de profesionalización docente -mostrar cómo se han formado en el tiempo las identidades profesionales en el mundo escolar-, o el de configuración de las culturas escolares, sin hacer referencia a los procesos de disciplinarización y academización de determinadas actividades o materias. La historia de la profesión docente y de las culturas escolares nos remite a la historia de las disciplinas y ésta, a su vez, a ambas.

Por otra parte, el auge en las últimas décadas del estudio de la cultura material de las instituciones educativas y de la enseñanza como actividad, así como del museísmo pedagógico y de la salvaguarda, conservación y estudio del patrimonio históricoeducativo ${ }^{3}$, ha abierto asimismo nuevas posibilidades a la historia de las disciplinas, actividades o materias, en especial, aunque no sólo, en relación en el ámbito científico-experimental. Durante la última década han proliferado en España los grupos y proyectos de investigación, encuentros, publicaciones y coloquios sobre la catalogación, conservación y estudio del patrimonio histórico-educativo. La confluencia, en este punto, con los historiadores de la ciencia y con el amplio movimiento a favor de la recuperación y catalogación del material científico de física, química y ciencias naturales, sobre todo -aunque no sólo- de los Institutos de Educación Secundaria llamados «históricos» -los creados a mediados del siglo XIX- ha hecho posible en España que este interés conservacionista e histórico se haya ampliado a otros elementos de la cultura escolar tales como los cuadernos, los trabajos de los alumnos, los exámenes, el mobiliario, los espacios escolares, los grabados y fotografías y, de un modo general, el material didáctico de todo tipo.

Más allá del uso nostálgico, del mero anticuariado o del fetichismo de los objetos utilizados en las instituciones educativas o producto de la cultura escolar, el análisis histórico de los mismos sólo cobra sentido en el marco de la historia de las disciplinas y actividades escolares. De ahí la confluencia, en este punto, de los historiadores de la ciencia con los de la educación en general y, más en concreto, de las didácticas específicas. Aspectos tales como la producción, comercialización y usos escolares de dichos objetos -entre ellos los manuales escolares- nos remiten de in-

3. Un buen ejemplo de este auge es la creación en el año 2003 de la Sociedad Española para el Estudio del Patrimonio Histórico-Educativo (SEPHE): http://www.institucional.us.es/paginasephe. mediato a la historia de determinadas disciplinas o actividades, y viceversa. Si, además, resulta que dichos objetos, y su historia, pueden ser utilizados actualmente -como de hecho está sucediendo en 
algún caso- como un recurso didáctico en la formación de profesores o en determinadas materias de diferentes niveles educativos, miel sobre hojuelas. ${ }^{4}$ Este es el mejor modo de demostrar su utilidad académica.

\section{Historia de las disciplinas y manualística escolar}

El interés académico y científico por los manuales escolares constituye en España -y fuera de ella- otra vía de promoción y apoyo al estudio de la historia de las disciplinas escolares, así como un campo privilegiado por los investigadores de dicha historia. La razón es obvia: dejando a un lado el fácil acceso a los mismos, los libros de texto han desempeñado -y siguen desempeñando- un papel clave en la fijación de los contenidos del código disciplinar de cada materia. Es en ellos donde se halla, junto a otros aspectos, el núcleo base, los contenidos y los enfoques fundamentales, de cualquier materia o disciplina. Hay, sin embargo, aspectos de su historia -en especial los relativos al libro de texto como producto comercial o a su regulación como tal producto- que sólo guardan, cuando la tienen, una relación indirecta o débil con la historia de las disciplinas o materias. No sucede lo mismo a la inversa: todos los aspectos de la historia de las disciplinas conciernen, en mayor o menor medida, a la de los libros de texto. Asimismo, como ya señaló Julia (2000), conviene prevenirse contra la idea de que la historia de una disciplina se reduce a la de los manuales utilizados en su enseñanza. 0 al contrario, añadimos, contra la idea de que es posible hacer la historia de una disciplina sin analizar la de sus libros de texto y el material empleado en su enseñanza. De un modo u otro, existe una relación estrecha entre ambas, aunque no igualitaria. Aun manteniendo, como campos de investigación, una relativa autonomía, la historia, el análisis de los libros de texto y del material de enseñanza como productos pedagógicos y culturales sólo adquiere un sentido histórico pleno cuando se inserta en el ámbito, más amplio, de la historia de las disciplinas.

Las consideraciones anteriores vienen a cuento de uno de los rasgos que, como se ha dicho, caracterizan la historiografía española sobre las disciplinas escolares: su desarrollo en los últimos años ha tenido lugar en buena parte dentro y a partir de los estudios sobre la historia de los manuales escolares. Por decirlo de un modo más claro: en más de un caso se ha llegado a la historia de las disciplinas -de una disciplinadesde la historia de sus manuales y libros de texto y no a la inversa. Basta hojear los índices de los libros publicados dentro o en la órbita del proyecto

4. Miel sobre hojuelas: dícese, en el habla popular española, de las cosas buenas que se ven favorecidas por otras aún mejores. 
MANES5 para advertir la presencia en los mismos de secciones o apartados dedicados a la historia de las disciplinas a través de sus manuales. Así, por ejemplo, de los veinticuatro trabajos incluidos en el volumen colectivo editado por Tiana (2000), catorce se agrupan bajo el epígrafe de "Los manuales escolares y la historia de las disciplinas»; en el libro, asimismo colectivo, dirigido por Guereña, Ossenbach y del Pozo (2005) figura un epígrafe específicamente titulado «Enfoques disciplinares»; y en el editado por Gómez García y Trigueros Gordillo (2000), sobre los manuales en la enseñanza secundaria, se dedica una última sección a «Las asignaturas y su evolución en los libros de texto». En igual sentido se manifiesta Valls (2001) en relación con un campo, la enseñanza de la historia, que es justamente aquél que cuenta, en comparación con otros, con un mayor número de investigaciones. Quizás haya llegado la hora de preguntarse si, manteniendo sus rasgos y aspectos propios, no sería conveniente insertar dichos estudios en el contexto de la historia de las disciplinas o, al menos, conectarlos a la misma. Es decir, si en vez de seguir analizando las disciplinas escolares a través de los libros de texto no sería más lógico y fructífero analizar los libros de texto desde la historia de las disciplinas, considerándolos como uno de los instrumentos fundamentales para la determinación y el conocimiento de la evolución del código disciplinar respectivo -junto, entre otros, con las regulaciones prescritas del currículum, las memorias y ejercicios de las oposiciones, los programas o programaciones, los cuadernos, apuntes y ejercicios de los alumnos y los exámenes-y, por tanto, en el contexto, más amplio, del proceso de disciplinarización de la materia o campo en cuestión.

\section{Algunos conceptos e ideas clave, de índole general, con los que estructurar la historia de las disciplinas}

La mayoría de los estudios de historia de las disciplinas se centran en un campo o área determinada, utilizan como fuente funda-

5. http://www.uned.es/manesvirtual/ProyectoManes/ proyecto.htm. En 1992 se lanzó en España el proyecto interuniversitario de investigación MANES con sede en la Universidad Nacional de Educación a Distancia, siguiendo la estela del proyecto Enmanuelle en Francia. Convertido dicho proyecto en Centro de Investigación MANES, su objetivo principal lo constituye hoy la investigación de los manuales escolares producidos en España, Portugal y América Latina. Sus coloquios, seminarios y publicaciones son sin duda una fuente indispensable para la historia de las disciplinas y un instrumento más para su configuración como un campo de investigación propio. mental los libros de texto y carecen de un aparato o marco conceptual y teórico explícito. Las líneas que siguen pretenden establecer las bases mínimas de dicho marco aplicables, por su generalidad, a cualquier campo o área disciplinar. Dichas bases facilitarían las comparaciones y la transferencia de resultados entre los estudios relativos a distintos campos disciplinares. 
Si las disciplinas pueden y deben ser consideradas como organismos vivos -nacen, evolucionan, se transforman, desaparecen, se fagocitan y engullen unas a otras, se desgajan, compiten, intercambian información, se aíslan, emparejan o forman tríos e incluso comunas, se atraen, se repelen o son indiferentes entre sí, tienen un nombre que las identifica, cambian de denominación y de apariencia, se jerarquizan, se hacen préstamos, se roban entre ellas, marcan su territorio: todo como en la vida mismaes posible hablar de un proceso de disciplinarización. Es decir, de conversión de una materia, de temas, de enfoques o de actividades en disciplinas académicas - 0 , a la inversa, de des-disciplinarización-, con avances, estancamientos y retrasos, en el que algo que no es todavía una disciplina, de modo pleno o según el modelo o tipo ideal, está en camino de serlo y puede ser calificado de protodisciplina o, como mucho, de proyecto de disciplina.

Lo primero que habría que deslindar y clarificar, pues, serían los conceptos de disciplina y de actividad, tarea o ejercicios. Lo segundo, dentro del proceso de disciplinarización, sería distinguir las diferentes fases que permitieran saber cuándo nos hallamos ante una protodisciplina o disciplina en germen y una disciplina como tal. Es decir, establecer cuáles son los elementos básicos que, de un modo más o menos sistemático u organizado, caracterizan una disciplina en el mundo académico.

Las disciplinas son, entre otras cosas, un producto más de las culturas escolares o, si se prefiere, de la gramática de la escolaridad. En este sentido, constituyen una modalidad más de las formas de hacer y pensar sedimentadas en el tiempo en forma de regularidades institucionales -en este caso, como asignaturas o campos de disciplinas afines- que conforman las culturas escolares. Como tales regularidades institucionales son, además, un producto social e histórico. Son el producto de un proceso de construcción como campos acotados que definen quiénes están fuera y quiénes están dentro. Un proceso en continua construcción resuelto, en sus diversas fases, mediante conflictos, negociaciones, acuerdos, componendas e imposiciones que reflejan las ortodoxias/heterodoxias en liza dentro de cada campo, y las estructuras y relaciones de poder académico existentes en el mismo. La presencia de mecanismos de control, por parte de quienes ocupan y acotan el campo disciplinar, sobre la formación y el acceso al mismo, y la configuración de un código disciplinar ortodoxo, constituyen dos de sus rasgos más relevantes.

El núcleo fundamental de toda disciplina escolar, lo que le distingue de otras y la configura como tal, es el código disciplinar. Dicho código, o tradición socialmente 
construida en confrontación o alianza con otras tradiciones disciplinares, está integrado por un conjunto de ideas, valores, suposiciones y pautas formales e informales, explícitas o implícitas, sobre: (a) un cuerpo de contenidos -saberes, conocimientos, destrezas, técnicas, habilidades- reconocible a través de memorias y ejercicios de oposiciones, libros de texto, programas, trabajos de alumnos y exámenes; (b) un discurso $o$ argumentos sobre su importancia y utilidad académica, profesional y laboral y su valor formativo que legitiman la presencia de la disciplina en los planes de estudio; y (c) unas prácticas y rutinas profesionales relativas tanto al modo de transmitir, enseñar y aprender los contenidos respectivos -lo que implica, entre otras cosas, analizar el contexto espacial y temporal en que la disciplina se imparte, sus útiles y materiales didácticos, y los roles y modos de interacción entre profesores y alumnoscomo presentarse en sociedad y en el mundo académico quienes las imparten (Cuesta, 1997 y 2003). La referencia final a las prácticas no es baladí. Nos alerta sobre la necesidad de distinguir entre la disciplina pretendida o soñada -la de las propuestas teóricas-, la regulada o prescrita -la de las normas legales- y la enseñada -la realidad- (Cuesta, 1998; Valls, 2001), a las que habría que añadir, la vivida y apropiada o hecha suya por los alumnos.

Por otra parte, las disciplinas no existen u operan en un espacio vacío, neutro o aislado. Surgen, anidan y se forman en el seno de sistemas educativos y de formas de organización de la escolaridad determinadas. Sólo se entienden en relación con otras disciplinas y campos en el contexto de dichos sistemas y formas. Su denominación, su lugar, su peso y su presencia en un determinado plan de estudios han de ser puestos en relación, desde una perspectiva diacrónica, con los de otras materias o disciplinas y con la sucesiva serie de reformas y modificaciones estructurales o curriculares del sistema educativo o modalidad de enseñanza de la que forman parte. De ahí la necesidad de que su estudio tenga en cuenta los procesos de sistematización, segmentación vertical (graduación) y horizontal (itinerarios y redes de centros diferenciadas), inclusión y exclusión (destinatarios) característicos de los sistemas educativos, así como las tendencias o fuerzas internas (presiones propedéuticas, establecimientos modelo, culturas escolares) que los conforman (Viñao, 2002).

Las disciplinas y campos disciplinares son por último, como se ha dicho, construcciones sociales y, por tanto, humanas. Es más, constituyen espacios académicos y escolares que existen gracias a que son ocupados por seres humanos que son los que les dan vida. El elemento humano, los profesores adscritos a un determinado 
campo o disciplina, no puede dejarse a un lado. 0, por ser más claros, otro aspecto fundamental de la historia de las disciplinas, sin el que no se entienden la formación y los cambios a lo largo del tiempo en los códigos disciplinares, es el de la historia de quienes las imparten y enseñan, de quienes las promueven y justifican y también, cómo no, de quienes ponen en cuestión su valor formativo y su existencia como tal disciplina académica.

Dicha historia, la de los profesores de una disciplina determinada -y la de los de otras disciplinas: no hay que olvidar nunca la visión que los demás tienen de uno mismo, el cómo nos ven otros-, abarca como mínimo la de su formación y titulaciones -¿cuáles poseían? ¿cuáles se exigían?-, los modos de selección -requisitos, concursos y oposiciones con sus memorias y criterios de valoración-, la carrera docente, el asociacionismo profesional, los grupos, escuelas y colegios visibles e invisibles que formaban, y su presencia y peso académico, social e institucional.

La historia de cualquier disciplina exige, pues, como tarea previa o paralela, una prosopografía de quienes componen el campo disciplinar. El diccionario de la Real Academia de la Lengua Española define la prosopografía como una "Descripción del exterior de una persona o de un animal". Su etimología nos remite al griego clásico: "prosopón" (aspecto) y "grafo" (describir). Sin embargo, el uso del término se ha generalizado en el campo de la historia para referirse a los estudios biográficos de todos o casi todos los componentes de un grupo social específico -por lo general profesional o político- con el fin de determinar y analizar no sólo sus características cuantitativamente mensurables, sino también, cada vez más, la urdimbre o tejido de relaciones e interacciones sociales generadas en el interior de dicho grupo. Relaciones e interacciones que contribuyen a diferenciarlo de otros -lo que permite saber quién está dentro o fuera del mismo-, a definirlo como tal grupo y a articular, en su interior, toda una serie de subgrupos, diferenciados entre sí pero en un continuo intercambio y movimiento de personajes, alianzas, conflictos, influencias y transvases de información y modos de actuación. De ahí la importancia, en la historia de las disciplinas, de las historias de vida y profesionales de quienes las impartieron, así como de relacionar la construcción social de las mismas con el proceso de profesionalización de sus docentes -la genealogía de la docencia como profesión en general y de cada categoría o cuerpo docente en particular-, y al menos en España, como en otros países, con el de la configuración de los cuerpos estatales de profesores. 


\section{Referencias bibliográficas}

CASPARD, Pierre. Introduction. Histoire de l'Éducation, Paris, n.38, maio de 1988, p.3-6.

CUESTA, Raimundo. Sociogénesis de una disciplina escolar: la Historia. Barcelona: Pomares-Corredor, 1997.

CUESTA, Raimundo. Clío en las aulas: la enseñanza de la Historia en España entre reformas, ilusiones y rutinas. Madrid: Akal, 1998.

CUESTA, Raimundo. Campo profesional, formación del profesorado y apuntes de didáctica crítica para tiempos de desolación. Didáctica de las Ciencias Experimentales y Sociales, Valencia, n. 17, p. 3-23, 2003.

GÓMEZ GARCÍA, Nieves; TRIGUEROS GODILLO, Guadalupe (eds.). Los manuales de texto en la enseñanza secundaria (1812-1990). Sevilla: Kronos, 2000.

GUEREÑA, Jean-Louis; OSSENBACH, Gabriela; DEL POZO, M. del Mar (eds.). Manuales escolares en España, Portugal y América Latina (siglos XIX-XX). Madrid: Uned, 2005.

JULIA, Dominique. La construcción de las disciplinas escolares en Europa. In: RUIZ BERRIO, Julio (ed.). La cultura escolar en Europa: tendencias históricas emergentes. Madrid: Biblioteca Nueva, 2000.

TIANA, Alejandro (ed.). El libro escolar, reflejo de intenciones políticas e influencias pedagógicas. Madrid: Uned, 2000.

VALLS, Rafael. La historiografía escolar española en la época contemporánea: de los manuales de historia a la historia de la disciplina escolar. In: FORCADELL, Carlos; PEIRÓ, Ignacio (coords.). Lecturas de la Historia: nueve reflexiones sobre Historia de la Historiografía. Zaragoza: Institución «Fernando el Católico», 2001.

VIÑAO, Antonio. Sistemas educativos, culturas escolares y reformas: continuidades y cambios. Madrid: Morata, 2002. Traducción al portugués: Sistemas educativos, culturas escolares e reformas. Mangualde: Edições Pedago, 2007.

VIÑAO, Antonio. El libro de texto y las disciplinas escolares. Una mirada a sus orígenes. In: ESCOLANO BENITO, Agustín (ed.). Currículum editado y sociedad del conocimiento: texto, multimedialidad y cultura de la escuela. Valencia: Tirant lo Blanch, 2006a.

VIÑAO, Antonio. Historia de las disciplinas escolares. Salamanca: Historia de la Educación, n. 25, p. 243-269, 2006b. Traducido al portugués y publicado en Revista Brasileira de História da Educação, São Paulo, n. 18, p. 173-215, 2008.

Recebido em 29 de março de 2012 e aprovado em 11 de maio de 2012. 
